\title{
Values of thoracic contrast-enhanced computed tomography in detecting incidental pulmonary thromboembolism in patients with malignant tumors
}

\author{
LIJUAN DAI, GAOFENG SHI, YANG LI and BO ZHAO \\ Department of Radiology, The Fourth Hospital of Hebei Medical University, Shijiazhuang, Hebei 050000, P.R. China
}

Received April 2, 2018; Accepted October 4, 2018

DOI: $10.3892 / \mathrm{ol} .2018 .9578$

\begin{abstract}
This study aimed to evaluate the values of thoracic multi-slice spiral computed tomography (CT) in the diagnosis of incidental pulmonary thromboembolism (IPTE) in patients with malignant tumors. The clinical data and imaging features of a total of 1,684 patients with malignant tumors, treated in the Fourth Hospital of Hebei Medical University, were analyzed retrospectively in order to investigate the types of malignancies, the patients' clinical features, and the emboli-preferred sites. Among the 1,684 patients, 60 patients had experienced IPTE (3.56\%), 35 were females (58.33\%), 25 were males (41.67\%). Lung cancer had the highest incidence $(n=22,36.67 \%)$. The most common site of IPTE was the left lower lobe of pulmonary artery $(n=46,76.67 \%)$. The imaging results revealed that in 5 patients $(8.33 \%)$ IPTE did not occur. Thoracic multi-slice spiral $\mathrm{CT}$ has a high detection rate of IPTE in patients with malignant tumors. Early diagnosis is helpful for early clinical treatment and has significant importance for patients' prognosis.
\end{abstract}

\section{Introduction}

Pulmonary thromboembolism (PTE) has a high incidence and mortality and accounts for $10-15 \%$ of adult deaths (1-3). The causes of PTE are complex and diverse. Patients with malignant tumors have greater probability to experience PTE due to the fact that the risk of thrombosis caused by braking, surgery, radiotherapy, chemotherapy, or metastasis in such patients is 4-6 times higher than that in normal populations $(2,3)$. It has been reported that the occurrence of incidental pulmonary thromboembolism (IPTE) found by conventional computed tomography (CT) in patients with malignant tumors has increased from 1.9 to $4.4 \%(4,5)$. The prognosis of malignant

Correspondence to: Dr Gaofeng Shi, Department of Radiology, The Fourth Hospital of Hebei Medical University, 12 Jiankang Road, Shijiazhuang, Hebei 050000, P.R. China

E-mail: dea3gx@163.com

Key words: multi-slice spiral computed tomography, malignancy, pulmonary embolism tumors with PTE is even worse. Timely and accurate diagnosis of IPTE has great impact on the post-treatment of patients with malignant tumors (6), is conducive to as-early-as-possible clinical treatment of such patients, and can improve their prognosis. The diagnosis of the PTE patients with obvious clinical symptoms is relatively simple, and the imaging diagnosis is based on CT pulmonary angiography (CTPA), but it is not easy for patients with mild or no clinical symptoms. Most cases have been found by chest enhanced CT. It has been reported that the false-negative rate of conventional CT for PTE in tumor patients ranges from 30 to $75 \%(7,8)$. Therefore, we should pay much attention to identify whether there is PTE in the conventional chest CT scan in patients with malignant tumors, especially when no clinical symptoms are present. This study analyzed the results of thoracic contrast-enhanced CT in order to research the incidence, type of malignant tumors, preferred site, and possible risk factors of IPTE in cancer patients with non-specific clinical signs and symptoms.

\section{Materials and methods}

Subjects. A total of 1,702 cancer patients who underwent multi-slice spiral CT in the Fourth Hospital of Hebei Medical University (Shijiazhuang, China) from January 2015 to February 2017 were reviewed, among whom 1,684 patients that met the inclusion criteria were included. The age range of the patients was 28-81 years old, with an average of $65.75 \pm 4.35$ years. Exclusion criteria: i) partial pulmonary arteries could not be traced due to various circumstances, such as severe pleural effusion, lung parenchymal mass, lung surgery history, or poor pulmonary vascular contrast agent filling; ii) patient had previous history of PTE, pulmonary mass, or metastatic lesions invading the pulmonary arteries; and iii) if for one patient multi-time scans were performed, only one scan was included. Patients with severe liver, kidney, or heart dysfunction; or allergic to iodine-containing agents were all excluded. This study was conducted in accordance with the Declaration of Helsinki and was approved by the Ethics Committee of the Fourth Hospital of Hebei Medical University. Written informed consent was obtained from all participants and/or guardians.

Methods (imaging protocol). All patients were scanned using Philips 256-slice Brilliance iCT (standard tube voltage, 


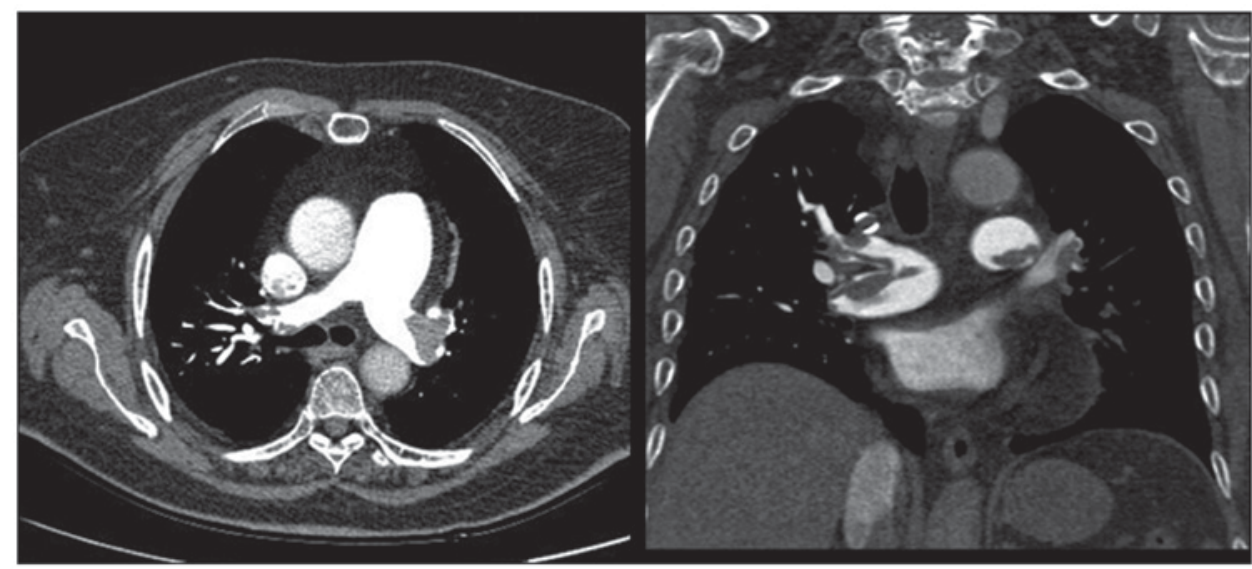

Figure 1. Axial and coronal views of thoracic contrast-enhanced CT. Left and right PTE is displayed in males, aged 45 years, with no clinical symptoms. CT, computed tomography; PTE, pulmonary thromboembolism.

$120 \mathrm{KV}$; tube current, automatic trigger tube current; scanning layer thickness, $0.9 \mathrm{~mm}$; layer spacing, $0.4 \mathrm{~mm}$; and collimation, $128 \pm 0.625 \mathrm{~mm}$; Philips Medical Systems B.V., Holland, The Netherlands). One dual-tube high-pressure syringe (Medrad, Inc.; Bayer HealthCare, Indianola, PA, USA) was used to inject the non-ionic contrast agent (Iopromide, 300 mg/ml; 1.5 ml/kg; Yangtze River Pharmaceutical Group, Ltd., Jiangsu, China) and $20 \mathrm{ml}$ of physiological saline via the ulnar vein. Each patient was inspected in the supine position with a venous injection speed of $4 \mathrm{ml} / \mathrm{sec}$. The scanning range was from the thoracic entrance to the diaphragm level.

Image post-processing. All images were reconstructed using the Adaptive Iterative Reconstruction Algorithm (ASIR). The lung images were then exhibited multi-directionally after multiplanar reformation, including the coronal and sagittal recombination and the maximum density projection (MIP). The positive case was defined as the detection of low-density filling defects in the main pulmonary arterial stem and its left and right pulmonary artery branches. The sites of the emboli and the accompanied abnormal pulmonary parenchymal signs were recorded.

\section{Results}

Cancer types. Among the 1,684 patients enrolled in this study, $1,026(60.93 \%)$ were females and $658(39.07 \%)$ were males. The age of the patients ranged from 28 to 81 years old, with an average of $65.75 \pm 4.35$ years old. The main tumor types were as follows: 438 cases of lung cancer (26\%), 256 cases of breast cancer (15.2\%), and 324 cases of gastrointestinal cancer (19.24\%); other tumor types included: liver cancer, pancreatic cancer, thymoma, gallbladder cancer, kidney cancer, endometrial cancer, colorectal cancer, and bone and soft tissue malignancies. Among the 1,684 patients, 60 patients $(3.56 \%)$ experienced IPTE. Their age ranged from 28 to 79 years old, with an average of $62.12 \pm 2.15$ years. Among these 60 patients with IPTE, 35 were females $(58.33 \%)$ and 25 were males (41.67\%), and the most common tumor types were: 22 cases of lung cancer $(36.67 \%), 7$ cases of gastric cancer $(11.67 \%)$, 6 cases of breast cancer (10\%), 4 cases of lymphoma $(6.67 \%)$, 3 cases of esophageal cancer (5\%), 3 cases of ovarial cancer
Table I. Major tumor types in the 60 patients with IPTE.

\begin{tabular}{lcc}
\hline Tumor type & $\mathrm{n}$ & Percentage \\
\hline Lung cancer & 22 & 36.67 \\
Gastric cancer & 7 & 11.67 \\
Breast cancer & 6 & 10 \\
Lymphadenoma & 4 & 6.67 \\
Esophageal cancer & 3 & 5 \\
Ovarial cancer & 3 & 5 \\
\hline
\end{tabular}

IPTE, incidental pulmonary thromboembolism.

(5\%), 2 cases of thymic carcinoma, gallbladder carcinoma, kidney cancer, liver cancer, bone and soft tissue malignant tumor, pancreatic cancer, endometrial carcinoma, respectively, and 1 case of colorectal cancer. The major tumor types in the patients with IPTE are shown in Table I. The tumor type associated with the highest incidence of IPTE is lung cancer (Table I and Fig. 1).

Sites of IPTE. A total of 148 emboli were found in the 60 IPTE patients, including 21 cases $(35 \%)$ at the right pulmonary arterial stem, 10 cases $(16.67 \%)$ at the left pulmonary arterial stem, 23 cases $(38.33 \%)$ at the right upper pulmonary arterial lobe and branches, 38 cases $(63.33 \%)$ at the right lower pulmonary arterial lobe and branches, 10 cases (16.67\%) at the left pulmonary arterial lobe and branches, and 46 cases (76.67\%) at the left lower pulmonary artery and branches. The most common site was the left lower pulmonary lobe and branches, and the rarest site was the pulmonary lingular segment of left upper pulmonary lobe. Among the 60 IPTE patients, 7 patients $(11.67 \%)$ had emboli in their lower extremity venous system, 1 patient $(1.6 \%)$ had emboli in the inferior vena cava, but 52 patients $(86.67 \%)$ were found with no embolus in the venous system. The imaging results revealed 55 IPTE cases (91.67\%), but 5 cases $(8.33 \%)$ were not revealed by the imaging results. The sites that were not detected with PTE included 2 cases at the posterior basal segment of right lower pulmonary lobe branches, 1 case at the lateral and posterior basal segment of 


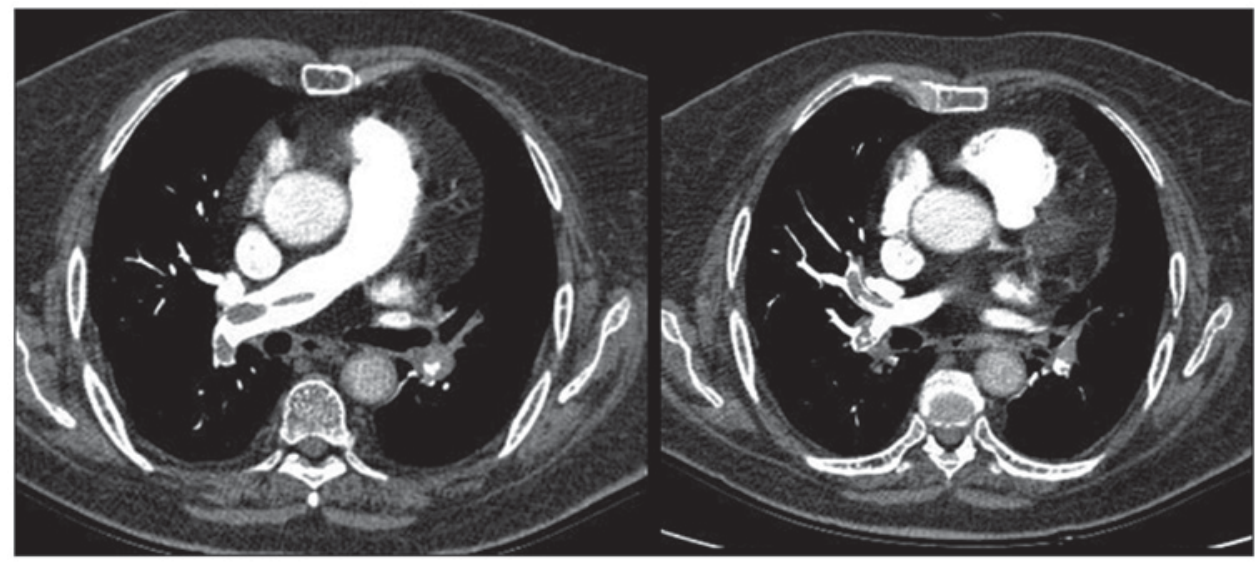

Figure 2. Axial views of thoracic contrast-enhanced CT. The PTE at the right pulmonary arterial stem and right lower pulmonary basilar arterial branches is displayed in males with history of liver cancer. CT, computed tomography; PTE, pulmonary thromboembolism.

Table II. Sites of the 148 emboli.

\begin{tabular}{lcc}
\hline Site & $\mathrm{n}$ & Percentage \\
\hline Right main pulmonary arterial stem & 21 & 35 \\
Left main pulmonary arterial stem & 10 & 16.67 \\
Right upper pulmonary arterial & 23 & 38.33 \\
lobe and branches & & \\
Right lower pulmonary arterial & 38 & 63.33 \\
lobe and branches & & \\
$\begin{array}{l}\text { Left upper pulmonary arterial } \\
\text { lobe and branches }\end{array}$ & 10 & 16.67 \\
Left lower pulmonary arterial & 46 & 76.67 \\
lobe and branches & & \\
\hline
\end{tabular}

left lower pulmonary lobe branches, respectively, and 1 case at the pulmonary lingular segment of left upper pulmonary lobe. The sites of IPTE are shown in Table II. Fig. 2 shows the PTE at the right pulmonary arterial stem and right lower pulmonary basilar arterial branches in males with history of liver cancer.

Complications. Among the 60 cancer patients with IPTE, 28 patients had a variety of complications, including 7 cases $(11.67 \%)$ of subacute atelectasis, 8 cases $(13.33 \%)$ of lung metastases, 6 cases (10\%) of pleural effusion, 2 cases $(3.33 \%)$ of mosaic perfusion area, and 5 cases $(8.33 \%)$ of pneumonia; but for the other 32 patients (53.33\%) no substantial changes were observed. Figs. 3 and 4 show a case of lung cancer accompanied by right middle lobe thromboembolism, right pleural effusion, and pericardial effusion; and a case of PTE at left lower pulmonary arterial lobe after breast cancer surgery.

\section{Discussion}

With the development of new multi-slice spiral CT technologies, the detection rate of thoracic contrast-enhanced CT in detecting PTE has been gradually increased. Advantages of these method include non-invasiveness, speed, and the capability to obtain images as far as sub-pulmonary arterial

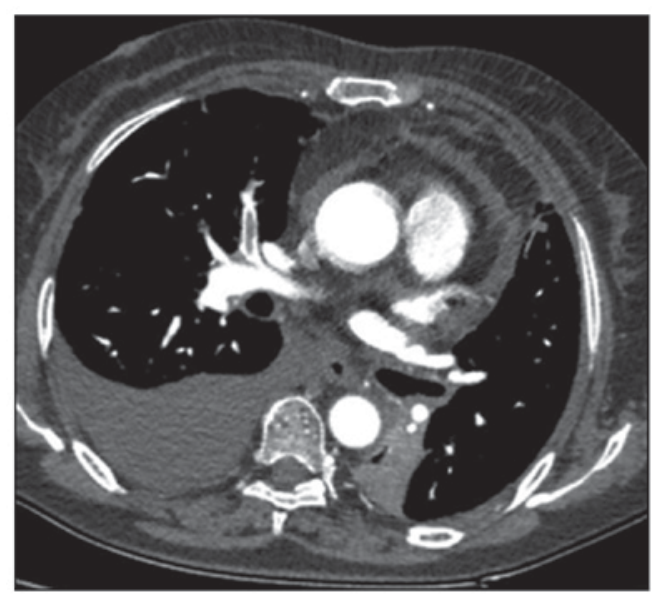

Figure 3. Lung cancer accompanied by right middle lobe thromboembolism, right pleural effusion, and pericardial effusion in female, aged 57 years.

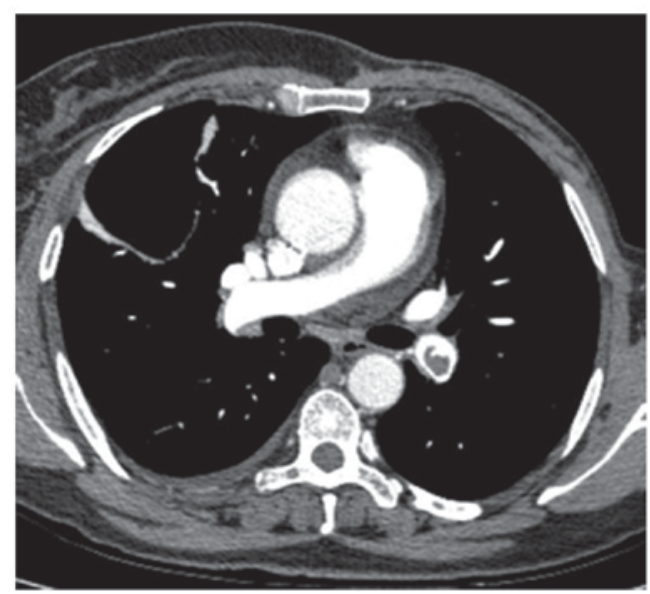

Figure 4. PTE at left lower pulmonary arterial lobe after breast cancer surgery in female, aged 56 years. PTE, pulmonary thromboembolism.

branches. At present, it is believed that in-time diagnosis of PTE in patients without specific clinical symptoms is very important, and therefore change of clinical treatment protocols is necessary in order to reduce mortality. It has been reported that the mortality of untreated PTE patients is $\sim 30 \%$, but this 
probability can be reduced to $3-10 \%$ by early diagnosis and appropriate treatment $(8,9)$. In this study, PTE was found to be more common in females than males, in consistency with a certain research (9). Several studies have reported that the incidence of IPTE in cancer patients is 2.5 or $4.6 \%$, respectively $(10,11)$. The incidence of IPTE in this study was found to be $3.56 \%$, similar to the reported results.

The mechanisms of IPTE in malignant tumors are different. In literature, it has been reported (12) that the malignancies that have the biggest probability of PTE are lung and colorectal cancer. Another study (13) has reported that the incidence of PTE is greater in breast cancer, lung cancer, and colon cancer, while it is rare in liver and cervical cancer. Another research has also reported that PTE occurs more often in tumors as lung cancer, malignant melanoma, and gynecological cancer, but the tumors with the lowest incidence of PTE are genitourinary malignancies (14). In this study, the patients with lung cancer had the highest probability of PTE, which is consistent with certain reports. We believe that lung cancer has the highest rate among all tumors, so its probability of IPTE is also the largest. Certain related studies have reported that in China, lung cancer has the highest incidence, so PTE in lung cancer accounts for the largest part $(15,16)$.

Previous findings show the sites of PTE to occur near the central and lobar pulmonary arteries (17). In this study, 108 emboli (72.97\%) were found in the proximal end of pulmonary artery (main stem and lobular segmental branch), and 40 emboli (27\%) occurred in the distal end (sub-segmental branch). In addition, another study evaluated the most common site for PTE to be the right pulmonary artery branch of lower lobe ( $\mathrm{n}=33,71.7 \%)$ (12). In this study, however, the incidence of PTE in the left lower pulmonary lobe was the highest, while the incidence was the lowest in the left upper pulmonary branch. The reason is not clear and may be related to the differences of the included cancer patients. In addition, thoracic contrast-enhanced CT can also observe the disease-caused conditions in the lung parenchyma, mediastinal pleura, or chest wall (5). The changes in the lung parenchyma associated with PTE include pulmonary infarction, pulmonary hemorrhage, mosaic perfusion, masses, atelectasis, or pleural effusion $(18,19)$. In this study, subarachnoid atelectasis occurred in 7 patients (11.67\%), pulmonary metastases occurred in 8 patients $(13.33 \%)$, pleural effusions were observed in 6 patients $(10 \%)$, mosaic perfusion area was observed in 2 patients (3.33\%), pneumonia occurred in 5 patients $(8.33 \%$ ), while for 32 patients $(53.33 \%)$ no substantial findings were observed. As for deep vein thrombosis, certain studies have reported that $34.4 \%$ of PTE patients have deep vein thrombosis $(20,21)$, and it has been reported that $43 \%$ of emboli originate from the deep venous system of the lower extremities, and 6.5\% originate from the inferior vena cava thrombosis $(22,23)$. The percentages in our study were 11.67 and $1.6 \%$, lower than those reported, and it is estimated to be related to the clinical treatment and patients' own conditions.

In conclusion, contrast-enhanced multi-slice spiral CT can improve the detection rate of IPTE in patients with no clinical symptoms, and the sites that are easy to be misdiagnosed should be carefully observed. Complications in PTE should be addressed for efficient early diagnosis and early treatment.
This study has some limitations. First, few cases are included in the study; thus, statistical errors may occur. Second, the cases included in this study are common malignant tumors in the region of Shijiazhuang, China. The incidence of malignant tumors in this region may be different from other regions, which may result in inconsistent results. Third, this study did not perform statistics toward the successful treatment, survival and mortality rate of the tumor patients with IPTE, which will be the aim of our future research.

\section{Acknowledgements}

Not applicable.

\section{Funding}

No funding was received.

\section{Availability of data and materials}

The datasets used and/or analyzed during the present study are available from the corresponding author on reasonable request.

\section{Authors' contributions}

LD drafted this manuscript. LD and GS were mainly devoted to collecting and interpreting the data. YL and $\mathrm{BZ}$ were responsible for image postprocessing. All authors read and approved the final manuscript.

\section{Ethics approval and consent to participate}

The study was approved by the Ethics Committee of the Fourth Hospital of Hebei Medical University (Shijiazhuang, China). Signed written informed consents were obtained from the patients and/or guardians.

\section{Patient consent for publication}

Not applicable.

\section{Competing interests}

The authors declare that they have no competing interests.

\section{References}

1. Connolly GC, Khorana AA, Kuderer NM, Culakova E, Francis CW and Lyman GH: Leukocytosis, thrombosis and early mortality in cancer patients initiating chemotherapy. Thromb Res 126: 113-118, 2010.

2. Geerts WH, Pineo GF, Heit JA, Bergqvist D, Lassen MR, Colwell CW and Ray JG: Prevention of venous thromboembolism: The Seventh ACCP Conference on Antithrombotic and Thrombolytic Therapy. Chest 126 (Suppl 3): 338S-400S, 2004.

3. Henzler T, Barraza JM Jr, Nance JW Jr, Costello P, Krissak R, Fink C and Schoepf UJ: CT imaging of acute pulmonary embolism. J Cardiovasc Comput Tomogr 5: 3-11, 2011.

4. Browne AM, Cronin CG, English C, NiMhuircheartaigh J, Murphy JM and Bruzzi JF: Unsuspected pulmonary emboli in oncology patients undergoing routine computed tomography imaging. J Thorac Oncol 5: 798-803, 2010. 
5. Dentali F, Ageno W, Becattini C, Galli L, Gianni M, Riva N, Imberti D, Squizzato A, Venco A and Agnelli G: Prevalence and clinical history of incidental, asymptomatic pulmonary embolism: A meta-analysis. Thromb Res 125: 518-522, 2010.

6. Cronin P, Weg JG and Kazerooni EA: The role of multidetector computed tomography angiography for the diagnosis of pulmonary embolism. Semin Nucl Med 38: 418-431, 2008.

7. Rossi SE, Goodman PC and Franquet T: Nonthrombotic pulmonary emboli. AJR Am J Roentgenol 174: 1499-1508, 2000.

8. Blachere H, Latrabe V, Montaudon M, Valli N, Couffinhal T, Raherisson C, Leccia F and Laurent F: Pulmonary embolism revealed on helical CT angiography: Comparison with ventilation-perfusion radionuclide lung scanning. AJR Am J Roentgenol 174: 1041-1047, 2000.

9. Farrell C, Jones M, Girvin F, Ritchie G and Murchison JT: Unsuspected pulmonary embolism identified using multidetector computed tomography in hospital outpatients. Clin Radiol 65 $1-5,2010$.

10. Ritchie G, McGurk S, McCreath C, Graham C and Murchison JT: Prospective evaluation of unsuspected pulmonary embolism on contrast enhanced multidetector CT (MDCT) scanning. Thorax 62: 536-540, 2007.

11. Lyman GH, Khorana AA, Kuderer NM, Lee AY, Arcelus JI, Balaban EP, Clarke JM, Flowers CR, Francis CW, Gates LE, et al; American Society of Clinical Oncology Clinical Practice: Venous thromboembolism prophylaxis and treatment in patients with cancer: American Society of Clinical Oncology clinical practice guideline update. J Clin Oncol 31: 2189-2204, 2013.

12. den Exter PL, van der Hulle T, Hartmann IJ, Jiménez D, Klok FA Huisman MV and Kroft LJ: Reliability of diagnosing incidental pulmonary embolism in cancer patients. Thromb Res 136 531-534, 2015.

13. Deniz MA, Deniz ZT, Adin ME, Akıl F, Turmak M, Urakc1 Z, Cetincakmak MG and Goya C: Detection of incidental pulmonary embolism with multi-slice computed tomography in cancer patients. Clin Imaging 41: 106-111, 2017.

14. Heyes GJ, Tucker A, Michael AL and Wallace RG: Erratum to: The incidence of deep vein thrombosis and pulmonary embolism following cast immobilisation and early functional bracing of Tendo Achilles rupture without thromboprophylaxis. Eur J Trauma Emerg Surg 41: 277, 2015.
15. Blom JW, Doggen CJ, Osanto S and Rosendaal FR: Malignancies, prothrombotic mutations, and the risk of venous thrombosis. JAMA 293: 715-722, 2005.

16. Shinagare AB, Guo M, Hatabu H, Krajewski KM, Andriole K, Van den Abbeele AD, DiPiro PJ and Nishino M: Incidence of pulmonary embolism in oncologic outpatients at a tertiary cancer center. Cancer 117: 3860-3866, 2011.

17. van Es N, Bleker SM and Di Nisio M: Cancer-associated unsuspected pulmonary embolism. Thromb Res 133 (Suppl 2): S172-S178, 2014.

18. Heit JA, Silverstein MD, Mohr DN, Petterson TM, O'Fallon WM and Melton LJ III: Risk factors for deep vein thrombosis and pulmonary embolism: A population-based case-control study. Arch Intern Med 160: 809-815, 2000.

19. McLaughlin PD, Liang T, Homiedan M, Louis LJ, O'Connell TW, Krzymyk K, Nicolaou S and Mayo JR: High pitch, low voltage dual source CT pulmonary angiography: Assessment of image quality and diagnostic acceptability with hybrid iterative reconstruction. Emerg Radiol 22: 117-123, 2015.

20. Wang H, Huang Y, Xu CW and Lin L: Clinical analysis of tumor and non-tumor patients complicated with pulmonary embolism. Int J Clin Exp Med 8: 18729-18736, 2015.

21. van der Hulle T, den Exter PL, Planquette B, Meyer G, Soler S, Monreal M, Jiménez D, Portillo AK, O'Connell C, Liebman HA, et al: Risk of recurrent venous thromboembolism and major hemorrhage in cancer-associated incidental pulmonary embolism among treated and untreated patients: A pooled analysis of 926 patients. J Thromb Haemost 14: 105-113, 2016.

22. Nagy Z, Horváth O, Kádas J, Valtinyi D, László L, Kopper B and Blaskó G: D-dimer as a potential prognostic marker. Pathol Oncol Res 18: 669-674, 2012

23. Spirk D, Aujesky D, Stuck AK, Beer JH, Mazzolai L, Baldi T, Banyai M, Hayoz D, Kaeslin T, Korte W, et al: Clinical outcomes of venous thromboembolism in patients with and without cancer: The SWIss Venous ThromboEmbolism Registry (SWIVTER). Semin Thromb Hemost 42: 642-649, 2016.

This work is licensed under a Creative Commons

Attribution-NonCommercial-NoDerivatives 4.0 International (CC BY-NC-ND 4.0) License. 